\title{
Retrospective Study of the Effects of Post-nasal Drip Symptoms on Cough Duration
}

\author{
TAKEO NAKAJIMA $^{1}$, TATSUYA NAGANO ${ }^{2}$ and YOSHIHIRO NISHIMURA ${ }^{2}$ \\ ${ }^{1}$ Nakajima Medical Clinic, Kobe, Japan; \\ ${ }^{2}$ Division of Respiratory Medicine, Department of Internal Medicine \\ Kobe University Graduate School of Medicine, Kobe, Japan
}

\begin{abstract}
Background/Aim: The aim of this study was to elucidate the significance of allergic rhinitis and post-nasal drip symptoms in patients with cough-variant and coughpredominant asthma. Patients and Methods: We conducted a retrospective analysis of 91 patients who had cough-variant or cough-predominant asthma and first visited the Nakajima Medical Clinic in Japan between June 2012 and July 2015. Results: Post-nasal drip symptoms were reported in 58 (63.7\%) patients. The patients with post-nasal drip symptoms (19.5 \pm 8.3 days) had a significantly longer time until cough disappearance than those without post-nasal drip symptoms (11.0 \pm 4.8 days) $(p=0.000034)$. Multivariate analysis showed that post-nasal drip symptoms are independent prolonged factors of cough duration. Conclusion: Post-nasal drip symptoms may affect cough control in patients with coughvariant and cough-predominant asthma.
\end{abstract}

Cough is the most common symptom worldwide and is caused by several diseases (1). Among these diseases, otolaryngological diseases are quite important in terms of cough treatment. For example, in Japan, $68.5 \%$ of patients with asthma have rhinitis, which is a cause of poor asthma control (2). Therefore, it is important to examine all patients with asthma for rhinitis. In the United States and Europe, post-nasal drip symptoms (PNDS) are an important condition causing cough as well as asthma (3). Cough due to postnasal drip is thought to be caused by stimulation of the

This article is freely accessible online.

Correspondence to: Tatsuya Nagano, MD, Ph.D., Assistant Professor of Division of Respiratory Medicine, Department of Internal Medicine, Kobe University Graduate School of Medicine, 7-5-1 Kusunoki-cho, Chuo-ku, Kobe, 650-0017, Japan. Tel: +81 783825660, Fax: +81783825661, e-mail: tnagano@med.kobeu.ac.jp

Key Words: Allergic rhinitis, post-nasal drip symptom, coughvariant asthma and cough-predominant asthma. sensory nerve ends of the hypopharynx and stimulation of the influx of post-nasal drip into the trachea, which activates the cough reflex pathway. The causes of PNDS are chronic sinusitis (39\%) and allergic rhinitis (AR) (23\%) (3). Cough caused by PNDS with AR is usually treated with antihistamines instead of antitussives.

Cough-variant asthma (CVA) is a subtype of asthma, and its symptoms include chronic cough without wheezing or dyspnea. CVA has almost normal lung function and mild bronchial hyperreactivity (BHR) and is sensitive to bronchodilators (4). CVA is a common cause of subacute and chronic cough in Japan, the United States, and the United Kingdom (5). The first-line therapy for CVA is based on inhaled corticosteroids (ICS). The use of ICS from the time of diagnosis of CVA can reduce the rate of transition to typical asthma (6). We reported that CVA led to wheezing in $30-40 \%$ of cases and transitioned to typical asthma within 1 year (7). Cough-predominant asthma (CPA) involves cough and mild wheezing, the former being the main symptom. The only difference between CVA and CPA is the presence (or absence) of mild wheezing; the clinical features and pathophysiology of the two are considered to be very similar. Moreover, the treatment strategy is also the same. Patients with CVA and CPA often have AR or PNDS (8). However, it is not clear how AR or PNDS affect the outcome of patients with CVA or CPA. Therefore, the aim of this study was to elucidate the significance of AR or PNDS in patients with CVA and CPA.

\section{Patients and Methods}

Participants. Ninety-one consecutive patients who visited Nakajima Medical Clinic with the chief complaint of cough lasting more than 3 weeks and diagnosed with CVA or CPA, were retrospectively analyzed. The diagnosis of AR was performed according to the Self Assessment of Allergic Rhinitis and Asthma (SACRA) questionnaire (9). This study was approved by the Review Board of the Hyogo Prefecture Medical Association (R1-012). This study was carried out by the opt-out method, and patients have a right not to participate in this study. 
Table I. Patient characteristics.

\begin{tabular}{lccc}
\hline & \multicolumn{2}{c}{ PNDS } & \\
\cline { 2 - 3 } & $\begin{array}{c}\text { Positive } \\
(\mathrm{n}=19)\end{array}$ & $\begin{array}{c}\text { Negative } \\
(\mathrm{n}=72)\end{array}$ & $p$-Value \\
& $5 / 14(26.3 \%)$ & $24 / 48(33.3 \%)$ & 0.78 \\
\hline Gender (male/female) & $36.5 \pm 11.3$ & $37.7 \pm 11.2$ & 0.68 \\
Age, years & $5.5 \pm 5.1$ & $6.8 \pm 9.2$ & 0.54 \\
Cough duration before & & & \\
visit to clinic, weeks & $3.54 \pm 0.93$ & $3.58 \pm 0.85$ & 0.88 \\
FVC (1) & $2.99 \pm 0.84$ & $3.01 \pm 0.77$ & 0.93 \\
FEV1 (1) & $84.3 \pm 6.0$ & $84.1 \pm 6.9$ & 0.94 \\
FEV1/FVC (\%) & $352.1 \pm 607.8$ & $227.7 \pm 503.7$ & 0.37 \\
Non-specific serum IgE & & & \\
(IU/ml) & $19 / 0(100 \%)$ & $39 / 33(54.2 \%)$ & 0.000079 \\
AR (yes/no) & $19 / 0(100 \%)$ & $72 / 0(100 \%)$ & 1.00 \\
Treated by ICS, ICS/LABA & & & \\
or SABA (yes/no) & & & \\
Treated by antihistamines & $6 / 13(31.6 \%)$ & $12 / 60(16.7 \%)$ & 0.19 \\
(yes/no) & & & \\
Treated by LTRA (yes/no) & $16 / 3(84.2 \%)$ & $47 / 25(65.3 \%)$ & 0.16 \\
Treated by intranasal & $9 / 10(47.4 \%)$ & $9 / 63(12.5 \%)$ & 0.0019 \\
corticosteroid (yes/no) & & & \\
Treated by prednisolone & $1 / 18(5.3 \%)$ & $4 / 68(55.6 \%)$ & 1.00 \\
(yes/no) & & & \\
\hline
\end{tabular}

PNDS: Post-nasal drip symptoms; FVC: forced vital capacity; FEV1: forced expiratory volume in 1s; FEV1/FVC: FEV1 as a percentage of FVC; AR: allergic rhinitis; ICS: inhaled corticosteroids; LABA: longacting $\beta 2$-agonist; SABA: short-acting $\beta 2$-agonist; LTRA: leukotriene receptor antagonist.

Diagnosis of CVA or CPA. We diagnosed CVA or CPA if all of the following conditions were met based on the 2012 guidelines of the Japanese Respiratory Society (https://minds.jcqhc.or.jp/docs/minds/ Cough/CPGs2012_Cough.pdf):

1. Cough without wheezing (on auscultation) that persists for more than 3 weeks ( 3 weeks).

2 . Bronchodilators ( $\beta$-stimulants or theophylline) are effective.

Exclusion criteria. The exclusion criteria were as follows: 1) obvious abnormal findings on chest radiography; 2) typical asthma; 3) smokers (including ex-smokers); 4) antitussives are effective; 5) clinical history of respiratory infection within 3 weeks; 6) cough after infection, gastroesophageal reflux disease (GERD), use of ACE inhibitors; 7) complications of sinusitis and usage of antibiotics from the first visit until cough disappearance; and 8) mild CVA or CPA in which cough disappeared only with $\beta 2$-agonist.

Study procedure. Fractional exhaled nitric oxide (FeNO) was measured using NIOX MINO ${ }^{\circledR}$ in all cases. The presence or absence of PNDS was investigated through interviews. We retrospectively analyzed the period from the start of treatment with inhaled corticosteroids (ICSs) or long-acting $\beta 2$-agonist (LABA) (medium or high dose) to the disappearance of cough, and the FeNO.

Evaluation of cough. The cough score was assessed via a canonical assessment method and calculated by first dividing the day into morning, noon, and night time periods, and scoring the cough
Table II. Multivariate analysis of factors that prolonged cough duration.

\begin{tabular}{lccc}
\hline & Hazard ratio & $\begin{array}{c}95 \% \text { confidence } \\
\text { interval }\end{array}$ & $p$-Value \\
\hline AR & 0.7723 & $0.5155-1.1570$ & 0.2100 \\
Antihistamine & 1.0060 & $0.6895-1.4670$ & 0.9800 \\
LTRA & 1.1760 & $0.8215-1.6840$ & 0.3800 \\
Prednisolone & 0.5397 & $0.2842-1.0250$ & 0.0590 \\
PNDS & 0.3791 & $0.2340-0.6141$ & 0.000081 \\
\hline
\end{tabular}

AR: Allergic rhinitis; LTRA: leukotriene receptor antagonist; PNDS: post-nasal drip symptoms.

frequency and intensity in each period to five levels from 0 to 4 . The scores of each period were summed, and the product of the frequency and strength was calculated (10). The point at which the cough score became 0 was defined as cough disappearance.

Statistical analysis. All data were entered into a pre-constructed Microsoft Excel sheet by the investigator (Takeo Nakajima). The entered data were checked by two authors and by a plausibility test during descriptive data analysis. All statistical analyses were performed with EZR version 1.37 (Saitama Medical Center Jichi Medical University; http://www.jichi.ac.jp/saitama-sct/SaitamaHP. files/statmed.html; Kanda, 2018), a graphical user interface for R (The R Foundation for Statistical Computing, Vienna, Austria, version 3.4.1) (11). Pearson's chi-squared test was used to detect possible associations between two categorical variables. The Mann-Whitney $U$-test was used to compare the differences between two independent samples. The Cox hazard model was used in multivariate analysis. $p$-Values $<0.05$ were considered statistically significant.

\section{Results}

Study participants. The characteristics of the study participants are summarized in Table I. PNDS were accompanied by CVA and CPA in 19 (20.9\%) patients. AR and nasal corticosteroids were significantly more frequent in patients with PNDS than in those without PNDS $(p=0.000079$ and 0.0036 , respectively). However, no significant differences were observed in the other characteristics.

FeNO. Although the FeNO level in the patients with AR $(35.6 \pm 25.5 \mathrm{ppb})$ tended to be higher than that in patients without $\operatorname{AR}(23.2 \pm 7.4 \mathrm{ppb})(p=0.134$, Figure 1$)$, there was no significant difference in FeNO levels in patients with PNDS $(35.9 \pm 27.2 \mathrm{ppb})$ compared to those without PNDS $(29.7 \pm 20.1$ ppb) ( $p=0.49$, Figure 1). This result suggested that FeNO is not effective for the diagnosis of PNDS in CVA or CPA.

Cough duration. Patients with AR (14.2 \pm 7.2 days) had a significantly longer time until cough disappearance than patients without AR (10.3 \pm 4.6 days) ( $p=0.0084$, Figure 2$)$. Furthermore, patients with PNDS (19.5 \pm 8.3 days) had a 

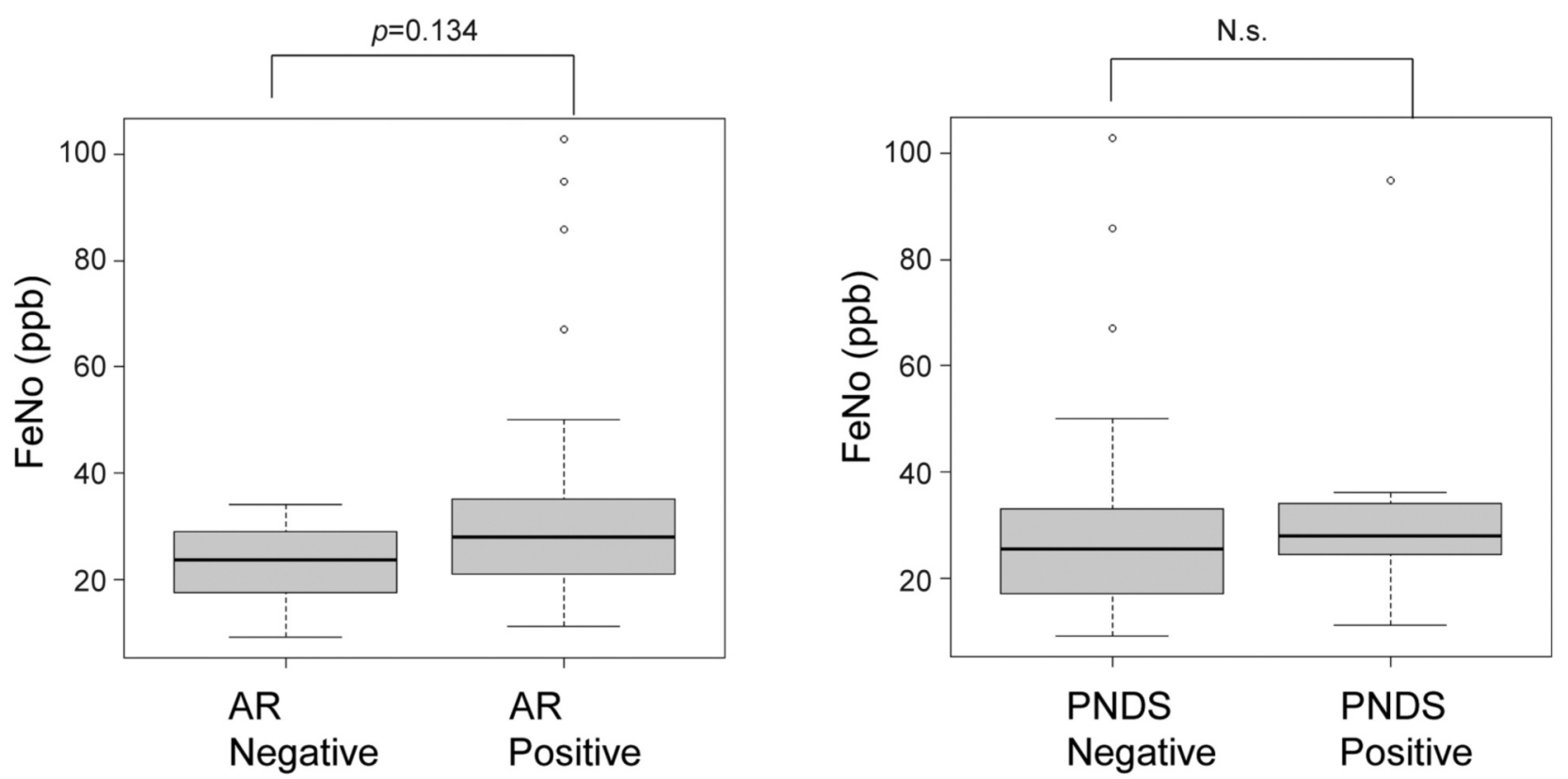

Figure 1. The concentration of fractional exhaled nitric oxide (FeNO). The levels of FeNO in patients with allergic rhinitis (AR) tended to be higher compared to that in patients without AR. The levels of FeNO in patients with post-nasal drip symptoms (PNDS) were compatible to those in patients without PNDS. Bars indicate means. N.s.: Not significant.
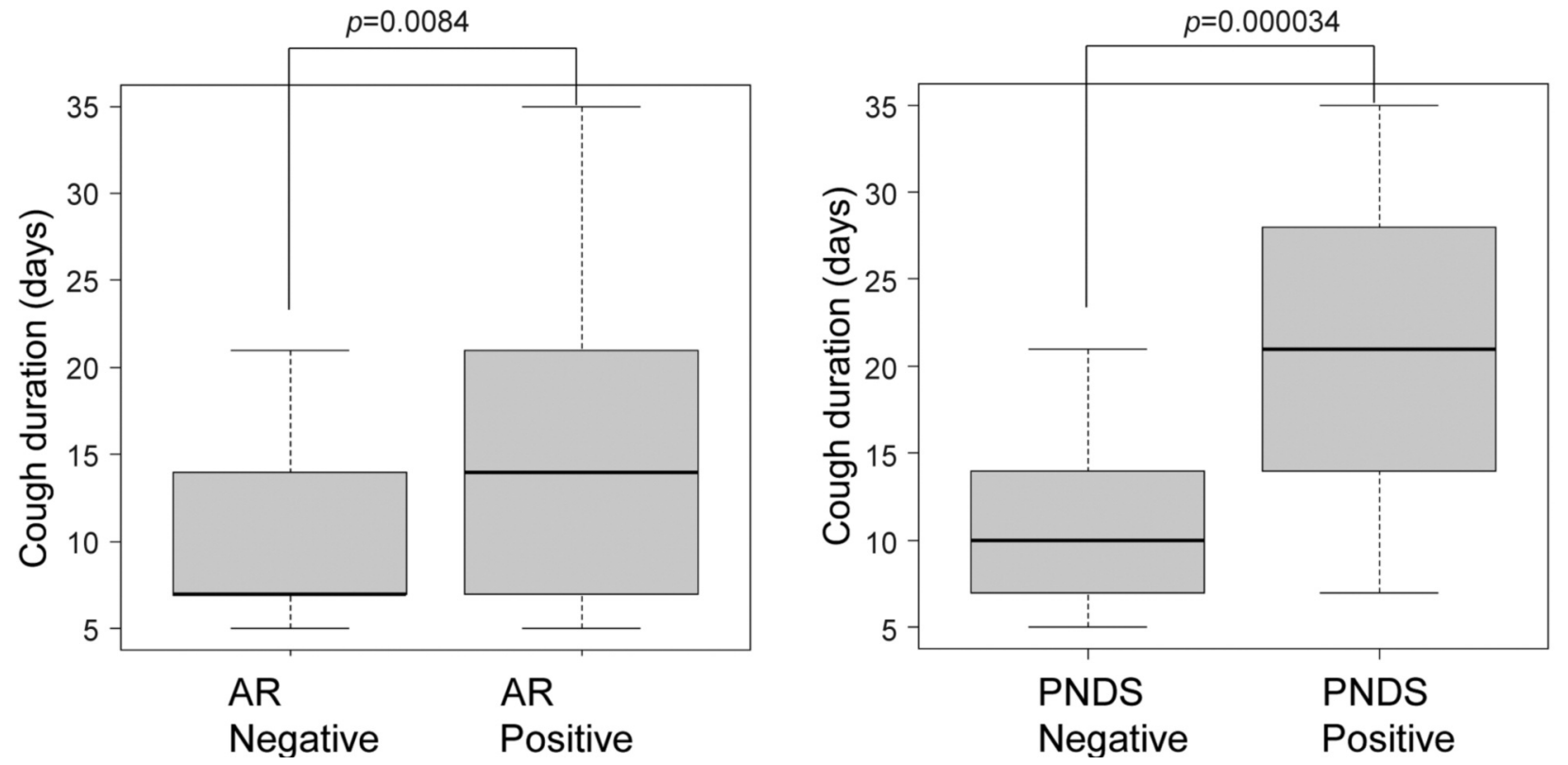

Figure 2. Duration from first visit to cough disappearance. Cough duration in patients with allergic rhinitis (AR) or post-nasal drip symptoms $(P N D S)$ was significantly longer than that in patients without AR $(p=0.0084)$ or PNDS $(p=0.000034)$, respectively. Bars indicate means.

significantly longer time until cough disappearance than those without PNDS (11.0 \pm 4.8 days) ( $p=0.000034$, Figure 2$)$. Multivariate analysis showed that PNDS were independent factors that prolonged cough duration (Table II).

\section{Discussion}

BHR in patients with CVA is mild to moderate compared to asthma (12-16). Therefore, some countries consider BHR to 
be important for the diagnosis of CVA. However, BHR can only be measured in a limited number of facilities in Japan. Since CVA is sensitive to $\beta$-stimulants (3), we adopted the diagnostic criteria used to identify CVA (cough for more than 3 weeks, no wheezing and efficacy of bronchodilators).

In the present study, there was no significant difference in FeNO levels among patients with and without PNDS. We considered that poor cough control due to PNDS may have less direct involvement of eosinophilic inflammation.

In the current study, we showed for the first time that AR and PNSD were independent prolonged factors of cough duration. Two double-blind clinical trials evaluating the efficacy of LTRA compared to placebo showed that LTRA was effective for CVA $(17,18)$. Two additional double-blind clinical trials evaluating the efficacy of antihistamine compared to placebo showed that LTRA was effective for CVA $(19,20)$. Prednisolone administration for 3 to 7 days is often used to treat severe cough. Therefore, we included LTRA, antihistamines, and prednisolone in the multivariate analysis.

In the present study, we did not show the efficacy of these drugs against CVA or CPA. However, this is a retrospective study, which is one of our limitations, and this type of study is not designed to evaluate drug efficacy. We could not draw any conclusions on the improvement brought about by these drugs on cough symptoms. Another limitation is that the patients were not seen by an otolaryngologist. In the literature, it is indicated that SACRA questionnaire-based AR treatment is effective for the detection of AR by respiratory physicians and for the offering of improved asthma control practices (21).

In conclusion, we showed that PNSD was independent prolonged factors of cough duration in patients with CVA or CPA. Further studies are needed to evaluate whether appropriate therapy against PNSD improves couch duration in patients with CVA or CPA.

\section{Conflicts of Interest}

The Authors state that they have no conflicts of interest in relation to this study.

\section{Authors' Contributions}

TakN and TatN wrote the manuscript. TakN made the database. TakN and YN conceived study design. TatN conducted the statistical analysis. All Authors analyzed the data, conceived the study, read and approved the final manuscript.

\section{Acknowledgements}

The Authors appreciate the members of the Division of Respiratory Medicine, Department of Internal Medicine, Kobe University Graduate School of Medicine for their helpful discussions. The
Authors would like to thank Editage (www.editage.com) for English language editing.

\section{References}

1 Otoshi T, Nagano T, Funada Y, Takenaka K, Nakata H, Ohnishi H, Nishiuma T, Nakajima T, Kageshita T, Tsuchiya T, Yamamoto M, Kobayashi K and Nishimura Y: A cross-sectional survey of the clinical manifestations and underlying illness of cough. In Vivo 33(2): 543-549, 2019. PMID: 30804139. DOI: 10.21873/ invivo. 11508

2 Ohta K, Bousquet PJ, Aizawa H, Akiyama K, Adachi M, Ichinose M, Ebisawa M, Tamura G, Nagai A, Nishima S, Fukuda T, Morikawa A, Okamoto Y, Kohno Y, Saito H, Takenaka H, Grouse L and Bousquet J: Prevalence and impact of rhinitis in asthma. SACRA, a cross-sectional nation-wide study in Japan. Allergy 66(10): 1287-1295, 2011. PMID: 21781135. DOI: 10.1111/j.1398-9995.2011.02676.x

3 Irwin RS, Curley FJ and French CL: Chronic cough.The spectrum and frequency of causes, key components of the diagnostic evaluation, and outcome of specific therapy. Am Rev Respir Dis 141(3): 640-647, 1990. PMID: 2178528. DOI: 10.1164/ajrccm/141.3.640

4 Corrao WM, Braman SS and Irwin RS: Chronic cough as the sole presenting manifestation of bronchial asthma. N Engl J Med 300(12): 633-637, 1979. PMID: 763286. DOI: 10.1056/ NEJM197903223001201

5 Niimi A, Ohbayashi H, Sagara H, Yamauchi K, Akiyama K, Takahashi K, Inoue H, Wakayama T, Kobayashi H, Hasegawa M, Kimura G, Yokoe T and Adachi M: Cough variant and cough-predominant asthma are major causes of persistent cough: a multicenter study in Japan. J Asthma 50(9): 932-937, 2013. PMID: 23841529. DOI: 10.3109/02770903.2013.823444

6 Matsumoto H, Niimi A, Takemura M, Ueda T, Tabuena R, Yamaguchi M, Matsuoka H, Hirai T, Muro S, Ito Y, Mio T, Chin $\mathrm{K}$, Nishiyama $\mathrm{H}$ and Mishima M: Prognosis of cough variant asthma: a retrospective analysis. J Asthma 43(2): 131-135, 2006. PMID: 16517429. DOI: 10.1080/02770900500498477

7 Nakajima T, Nishimura Y, Nishiuma T, Kotani Y, Funada Y, Nakata $\mathrm{H}$ and Yokoyama M: Characteristics of patients with chronic cough who developed classic asthma during the course of cough variant asthma: a longitudinal study. Respiration 72(6): 606-611, 2005. PMID: 16113512. DOI: 10.1159/000087459

8 Tajiri T, Niimi A, Matsumoto H, Ito I, Oguma T, Otsuka K, Takeda T, Nakaji H, Inoue H, Iwata T, Nagasaki T and Mishima M: Prevalence and clinical relevance of allergic rhinitis in patients with classic asthma and cough variant asthma. Respiration 87(3): 211-218, 2014. PMID: 24401902. DOI: 10.1159/000355706

9 Hojo M, Ohta K, Iikura M, Mizutani T, Hirashima J and Sugiyama $\mathrm{H}$ : Clinical usefulness of a guideline-based screening tool for the diagnosis of allergic rhinitis in asthmatics: the self assessment of allergic rhinitis and asthma questionnaire. Respirology 18(6): 10161021, 2013. PMID: 23668782. DOI: 10.1111/resp.12116

10 Miwa N, Nagano T, Ohnishi H, Nishiuma T, Takenaka K, Shirotani T, Nakajima T, Dokuni R, Kawa Y, Kobayashi K, Funada Y, Kotani Y and Nishimura Y: An open-label, multiinstitutional, randomized study to evaluate the additive effect of a leukotriene receptor antagonist on cough score in patients with cough-variant asthma being treated with inhaled corticosteroids. Kobe J Med Sci 64(4): E134-E139, 2018. PMID: 30728339. 
11 Kanda Y: Investigation of the freely available easy-to-use software 'EZR' for medical statistics. Bone Marrow Transplant 48(3): 452-458, 2013. PMID: 23208313. DOI: 10.1038/ bmt. 2012.244

12 Fujimura M, Kamio Y, Hashimoto T and Matsuda T: Cough receptor sensitivity and bronchial responsiveness in patients with only chronic nonproductive cough: in view of effect of bronchodilator therapy. J Asthma 31(6): 463-472, 1994. PMID: 7961323. DOI: $10.3109 / 02770909409089488$

13 Tokuyama K, Shigeta M, Maeda S, Takei K, Hoshino M and Morikawa A: Diurnal variation of peak expiratory flow in children with cough variant asthma. J Asthma 35(2): 225-229, 1998. PMID: 9576149. DOI: $10.3109 / 02770909809068211$

14 Niimi A, Amitani R, Suzuki K, Tanaka E, Murayama T and Kuze F: Eosinophilic inflammation in cough variant asthma. Eur Respir J 11(5): 1064-9, 1998. PMID: 9648956. DOI: 10.1183/ 09031936.98.11051064

15 Matsumoto H, Niimi A, Takemura M, Ueda T, Yamaguchi M, Matsuoka H, Jinnai M, Chin K and Mishima M: Features of cough variant asthma and classic asthma during methacholineinduced brochoconstriction: a cross-sectional study. Cough 9;5:3 2009. PMID: 19272144. DOI: 10.1186/1745-9974-5-3

16 Mochizuki H, Arakawa H, Tokuyama K and Morikawa A: Bronchial sensitivity and bronchial reactivity in children with cough variant asthma. Chest 128(4): 2427-2434, 2005. PMID: 16236905. DOI: $10.1378 /$ chest.128.4.2427

17 Dicpinigaitis PV, Dobkin JB and Reichel J: Antitussive effect of the leukotriene receptor antagonist zafirlukast in subjects with cough-variant asthma. J Asthma 39(4): 291-297, 2002. PMID: 12095178. DOI: $10.1081 /$ jas-120002285
18 Spector SL and Tan RA: Effectiveness of montelukast in the treatment of cough variant asthma. Ann Allergy Asthma Immunol 93(3): 232-236, 2004. PMID: 15478381. DOI: 10.1016/S1081-1206(10)61493-7

19 Shioya T, Ito N, Sasaki M, Kagaya M, Sano M, Shindo T, Kashima $M$ and Miura $M$ : Cough threshold for capsaicin increases by azelastine in patients with cough-variant asthma. Pulm Pharmacol 9(1): 59-62, 1996. PMID: 8843511. DOI: 10.1006/pulp.1996.0007

20 Shioya T, Satake M, Sano M, Kagaya M, Watanabe A, Sato K, Ito T, Ito N, Sasaki M and Miura M: Effect of suplatast tosilate, a Th2 cytokine inhibitor, on cough variant asthma. Eur J Clin Pharmacol 58(3): 171-176, 2002. PMID: 12107601. DOI: 10.1007/s00228-002-0468-Z

21 Yasuo M, Kitaguchi Y, Komatsu Y, Hama M, Koizumi T, Agatsuma T, Ichiyama T, Kato A, Moteki H and Hanaoka M: Self-assessment of allergic rhinitis and asthma (SACRA) questionnaire-based allergic rhinitis treatment improves asthma control in asthmatic patients with allergic rhinitis. Intern Med 56(1): 31-39, 2017. PMID: 28049997. DOI: 10.2169/ internalmedicine .56 .7251
Received January 28, 2021

Revised February 4, 2021

Accepted February 5, 2021 